\title{
Neurological manifestations of COVID-19: with emphasis on Iranian patients
}

\author{
Cynthia Aslan $^{1,2} \cdot$ Sepideh Nikfarjam ${ }^{3} \cdot$ Mohammad Asadzadeh $^{4} \cdot$ Reza Jafari $^{5}$ (D)
}

Received: 18 August 2020 / Revised: 16 February 2021 / Accepted: 24 February 2021 / Published online: 12 March 2021

(c) Journal of NeuroVirology, Inc. 2021

\begin{abstract}
The novel coronavirus disease 2019 (COVID-19) caused by the severe acute respiratory syndrome coronavirus 2 (SARS$\mathrm{CoV}-2$ ) has instigated a global pandemic as a formidable and highly contagious infectious disease. Although the respiratory system remains the most frequently affected organ, several case reports have revealed that the complications are not merely limited to the respiratory system, and neurotropic and neuroinvasive properties have also been observed, leading to neurological diseases. In the present paper, it was intended to review the possible neuroinvasive routes of SARS-CoV-2 and its mechanisms that may cause neurological damage. Additionally, the neurological manifestations of COVID-19 across the globe were discussed with emphasis on Iran, while highlighting the impact of SARS-CoV-2 on the central and peripheral nervous systems.
\end{abstract}

Keywords COVID-19 $\cdot$ Coronavirus $\cdot$ SARS-CoV-2 $\cdot$ Neurological manifestations $\cdot$ Iran

\section{Introduction}

The novel coronavirus disease 2019 (COVID-19) initiated from Wuhan in China, and since then, it has rapidly spread around the globe. The most prevalent sign in patients with

Reza Jafari

Jafari.reza@umsu.ac.ir

Cynthia Aslan

cynthia.aslan@ymail.com

Sepideh Nikfarjam

nikfarjams@tbzmed.ac.ir

Mohammad Asadzadeh

asadzadeh.m@ajums.ac.ir

1 Immunology Research Center, Tabriz University of Medical Sciences, Tabriz, Iran

2 Department of Immunology, Faculty of Medicine, Tabriz University of Medical Sciences, Tabriz, Iran

3 Department of Medical Biotechnology, Faculty of Advanced Medical Sciences, Tabriz University of Medical Sciences, Tabriz, Iran

4 Department of Radiology, School of Medicine, Ahvaz Jundishapur University of Medical Sciences, Ahvaz, Iran

5 Solid Tumor Research Center, Cellular and Molecular Medicine Research Institute, Urmia University of Medical Sciences, Urmia, Iran confirmed COVID-19 cases is the involvement of the respiratory system; however, apart from respiratory and systemic symptoms, there are several reports regarding the neurotropic properties of severe acute respiratory syndrome coronavirus 2 (SARS-CoV-2), the cause of COVID-19 (Conde et al. 2020). Although the exact pathway through which SARS-CoV-2 reaches the brain is not yet fully understood, two main routes appear to explain this neurological attack: peripheral nerves route and hematogenous spread (Baig et al. 2020; Montalvan et al. 2020; Netland et al. 2008). Valuable evidence regarding the SARS-CoV-2 neuroinvasiveness was found by animal models of SARS and MERS (the Middle East respiratory syndrome), both caused by coronaviruses (CoVs) of similar structure, SARS-CoV and MERS$\mathrm{CoV}$, during the 2002 and 2012 epidemics, respectively. All these pathogens exploit angiotensin-converting enzyme 2 (ACE2) to gain access to the target cells. The so-called CoVs fusion to their host cell membrane receptor, ACE2, is facilitated by CoV spike glycoprotein (Natoli et al. 2020). ACE2 receptors are distributed among the nervous system tissue, endothelial cells, and tissues of many other organs. Compared with previous $\mathrm{CoVs}$, the SARS-CoV-2 spike glycoprotein demonstrates a higher affinity toward the ACE2 receptor, and therefore, it may have higher neuroinvasive potential as well (Berger 2020). The present study aimed to review the possible mechanisms of SARS-CoV-2 neuroinvasion along 
with the major neurological signs and symptoms reported in patients with COVID-19 all over the world, particularly in Iran (Table 1).

\section{SARS-CoV-2}

The latest CoV, SARS-CoV-2, is classified as a beta-CoV that shares high genetic similarities $(79.5 \%)$ with SARSCoV (Ahmadirad and Ghasemi 2020; Conde et al. 2020). Both viruses employ spike proteins present on the virion surface to recognize and interact with ACE-2 expressed on mammalian host cells. Afterward, the transmembrane serine protease 2 (TMPRSS2) allows the virus to utilize ACE-2 as a receptor to gain entry inside the host cells (Zubair et al. 2020). Human ACE2 is highly expressed by airway epithelia, lung parenchyma, gastrointestinal system, kidneys, and blood vessels through the entire body and extensively throughout the central nervous system (CNS) (Chen et al. 2020). In the brain, ACE2 protein is generally found on glial cells and neurons (Gowrisankar and Clark 2016; $\mathrm{Xia}$ and Lazartigues 2010). Interestingly, it was reported that ACE2 has a 10-20-fold higher binding affinity toward the SARS-CoV-2 spike protein than that of the SARS-CoV (Wang et al. 2020). The question of how SARS-CoV-2 can reach the CNS is not yet answered; nonetheless, studies on humans and experimental models revealed that the CoVs access the CNS either via the neuronal retrograde or hematogenous route (Conde et al. 2020).

\section{Entry routes of SARS-CoV-2 to CNS}

\section{Neuronal retrograde route}

In this type of CNS invasion route, SARS-CoV-2 infects a peripheral neuron and then continues to infect other neurons by means of traveling through a retrograde transport system facilitated by dinein and kinesin proteins. After being released into the presynaptic terminal by exocytosis, the virus interacts with the existing ACE2 receptors on the postsynaptic neuron membrane to gain entry using the receptormediated endocytosis (Bohmwald et al. 2018).

\section{Olfactory pathway}

In the case of viruses that infiltrate the body via intranasal delivery, the olfactory pathway is an excellent mechanism to gain access to the brain (Swanson II and McGavern 2015). An olfactory neuron is in direct contact with the external environment with the gateway to the CNS, i.e., the olfactory bulb (Bohmwald et al. 2018; II and McGavern 2015). In a series of studies, rodent models were used for understanding the neuroinvasiveness of several CoVs via the so-called nerve route (Dubé et al. 2018; Netland et al. 2008). For instance, Netland et al. demonstrated that following the intranasal inoculation, $\mathrm{CoV}$ was detected after $60 \mathrm{~h}$ in the olfactory bulb and after 4 days in the piriform as well as the infralimbic cortex, basal ganglia, and the midbrain (Montalvan et al. 2020; Netland et al. 2008). Similar results were also reported for another type of CoV, human CoV OC43 (HCoV-OC43), in another study. In this inquiry, $\mathrm{CoV}$ was detected four days after inoculation in the piriform cortex, brain stem, and spinal cord (Dubé et al. 2018). Interestingly, another research from the 1990s indicated that once the olfactory pathway was interrupted, the transneuronal movement of the mouse hepatitis virus (MHV; a type of $\mathrm{CoV}$ ) was prevented in an animal model (Perlman et al. 1990). Furthermore, Jacomy et al. reported that nasal instillation of $\mathrm{HCoV}-\mathrm{OC} 43$ in $\mathrm{BALB} / \mathrm{c}$ and $\mathrm{C} 57 \mathrm{BL} / 6$ murine models resulted in a generalized CNS infection, representing the neuroinvasiveness and neurovirulence of HCoV-OC43 (Jacomy and Talbot 2003). Similar findings were also reported by St-Jean et al. using $\mathrm{HCoV}-\mathrm{OC} 43$ inhalation in mice. In this case, viral antigens were detected in the olfactory bulb by the third day of inhalation, and the virus was detected throughout all brain regions after seven days, evidently implying that it can rapidly replicate and cause fatal encephalitis once set in CNS (St-Jean et al. 2004). Glass et al. described that SARS-CoV could infect the bronchial and bronchiolar epithelial cells of the respiratory system, and eventually reach the brain and lead to a systemic nonlethal infection in B6 mice (Glass et al. 2004). A plethora of studies has revealed that the brainstem is among the severely infected areas, both in patients' and in experimental animals' brains. As the most characteristic symptom of COVID-19 patients is respiratory failure, it remains to determine whether the neuroinvasive potential of SARSCoV-2 plays a role in the patients' acute respiratory distress with COVID-19 (Li et al. 2020b).

\section{Gut-brain axis}

Accumulating evidence supports the theory that the intestines probably act as an "entrance door" through which viruses may either directly invade the brain or indirectly trigger immune responses in the enteric nervous system (ENS) and ascend to CNS through intestinal vagal afferents. The enteric glial cells (EGCs) function as antigenpresenting cells (APC) by providing the immune cells located in the gut-associated lymphoid tissue with viral antigenic material. A gut infection may prompt a peripheral immune response (e.g., an adverse cytokine storm) and promote enteric neuroinflammation (Esposito et al. 2017). Scientists suggest that SARS-CoV-2-associated diarrhea ought to be considered as gastrointestinal dysfunction that 


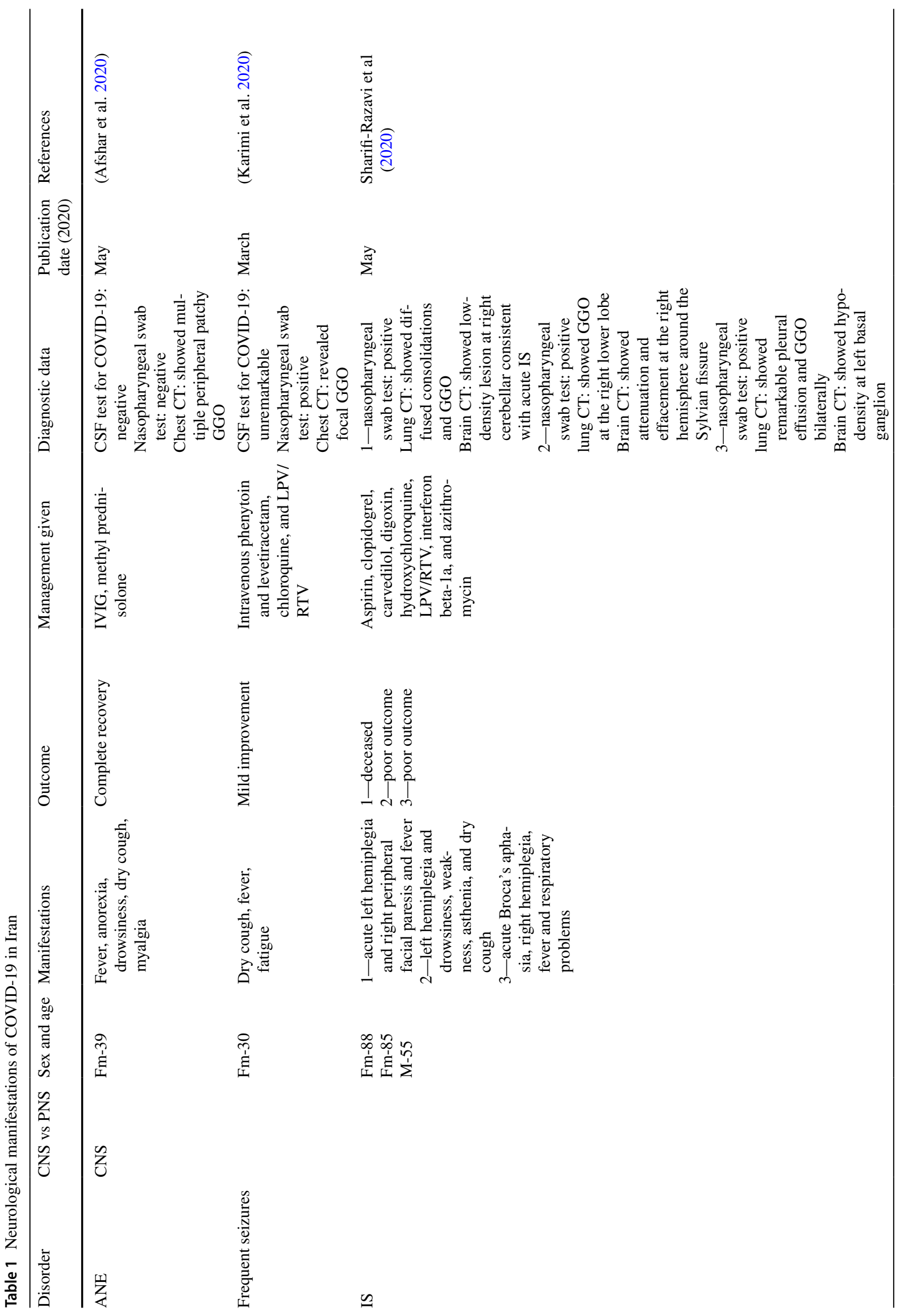




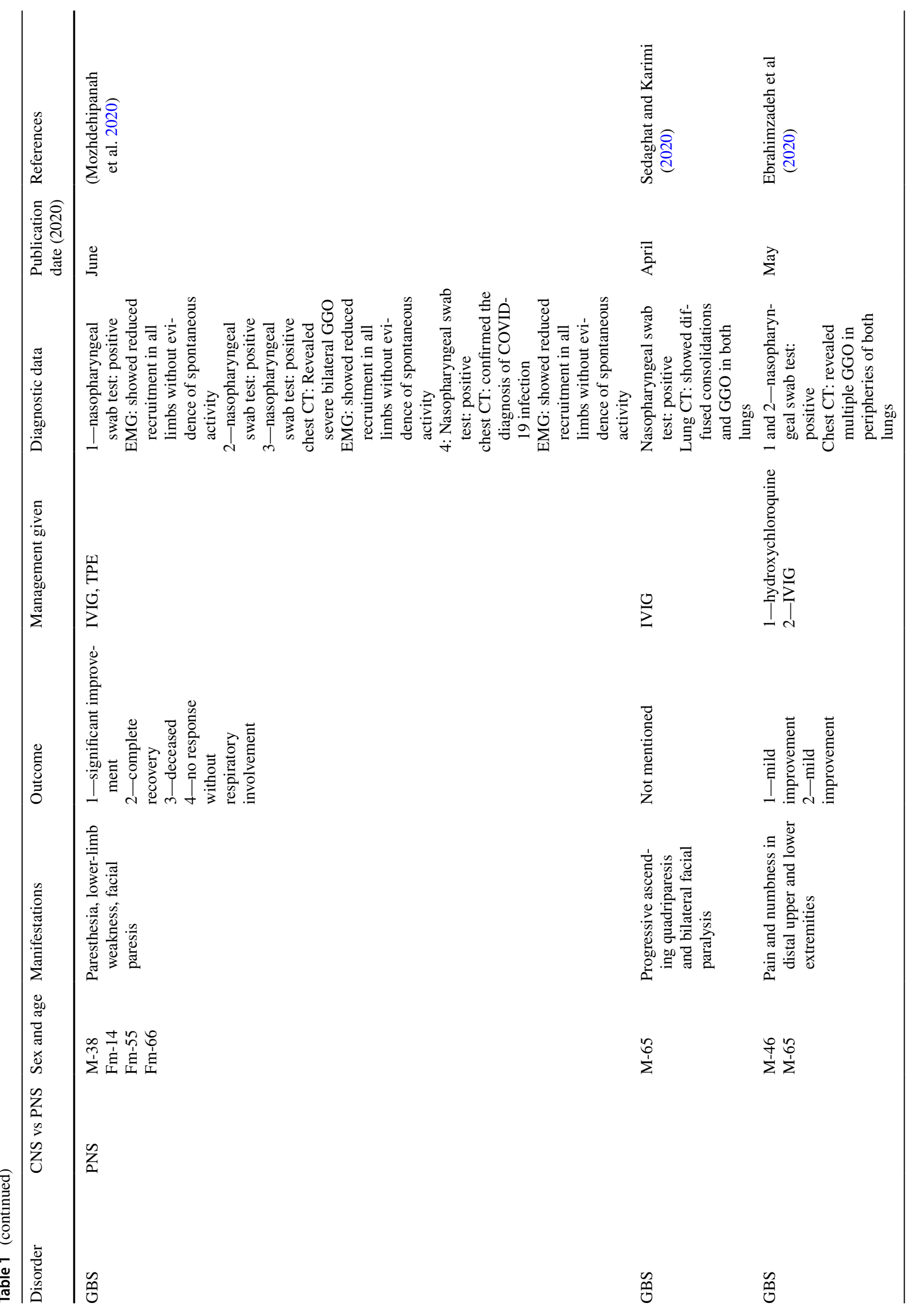


is a conceivable sign of ENS/EGC involvement, offering an alternative pathway for SARS-CoV-2 neuroinvasion (Esposito et al. 2020).

\section{Hematogeneic pathway}

\section{The blood-brain barrier}

For accessing the CNS through arteries, the virus must overcome the blood-brain barrier (BBB). Paniz-Mondolfi et al. found virion-like particles in capillary endothelial cells of the brain sprouting across the endothelium. Henceforth, the endothelial bed and the hematogenous pathway are introduced as the possible route for brain entrance of SARSCoV-2. The production of ACE2 by the capillary endothelial tissue also confirms the asserted hypothesis (Paniz-Mondolfi et al. 2020). Moreover, McCray et al. demonstrated that a consequence of enhanced cytokine and chemokine secretion in the lung tissue of K18-hACE2 mice compared with nontransgenic mice is the augmented permeability of BBB, which probably assists the wide-ranging delivery of SARSCoV into the CNS (McCray et al. 2007).

\section{Trojan horse mechanism}

Some viruses, such as the Zika virus and the human immunodeficiency virus (HIV), enforce leukocytes to transport them as cargo through the BBB instead of directly penetrating the $\mathrm{BBB}$, which is known as the "Trojan horse" mechanism (Huang et al. 2020; McGavern and Kang 2011). In other words, CoVs have the ability to employ leukocytes as vectors (Huang et al. 2020). One example is the HCoV-229E virus that infects monocytes/macrophages (Collins 2002; Desforges et al. 2007), dendritic cells, and peritoneal macrophages, and uses them as reservoir to induce chemokine secretion (Wentworth et al. 2005).

\section{Cellular and molecular mechanisms of CoV-mediated CNS damage}

Neuronal cell death following $\mathrm{CoV}$ infection has been documented in several reports. Lavi et al. demonstrated that MHV-A59 (an experimental murine coronavirus) is able to reach the brain via intranasal or intracerebral routes, and its neurovirulence is almost certainly facilitated by cytokines secreted from microglia and type I astrocytes. The pathogenesis of MHV-A59 infection is comparable to that of COVID19 (Lavi and Cong 2020). Wheeler et al. found that proinflammatory cytokines such as interleukin 6 (IL-6), IL-12 $\mathrm{p} 40$, IL-15, and tumor necrosis factor $\alpha$ (TNF- $\alpha)$ in comparison with a non-neurotropic MHV were increased in primary glial cultures of MHV-A59-infected cells. These findings suggest that infection with a neurotropic virus causes activation of glial cells and secretion of pro-inflammatory cytokines (Bohmwald et al. 2018; Wheeler et al. 2018). TNF- $\alpha$ secreted from activated microglial cells is also able to induce neuronal apoptosis. Jacomy et al. reported that $\mathrm{HCoV}-\mathrm{OC} 43$-infected cells release elevated levels of TNF- $\alpha$ in vitro, which is possibly involved in at least part of the apoptosis detected in nearby non-infected neurons, and might be responsible for CNS impairment in surviving animals (Jacomy et al. 2006).

\section{Neurological manifestation of COVID-19}

According to an increasing number of studies, a variety of neurological symptoms have been explained in COVID-19 patients. Neurological manifestations of COVID-19 are generally divided into two categories: CNS-related manifestations such as acute necrotizing encephalopathy (ANE) and ischemic stroke (IS), and the peripheral nervous system (PNS)-related manifestations such as Guillain-Barré syndrome (GBS) and myasthenia gravis (MG) (de Goés et al. 2020).

\section{CNS manifestations}

\section{Acute necrotizing encephalopathy}

Acute necrotizing encephalopathy (ANE) is an unusual brain condition that occurs following a viral infection associated with intracranial cytokine storms that lead to BBB collapse without direct invasion of virus or parainfectious demyelination (Poyiadji et al. 2020). ANE's initial presentations usually occur $12-72 \mathrm{~h}$ after the viral infection symptom onset comprised of seizures, reduced consciousness, and vomiting (Dixon et al. 2020). The first presumptive case of COVID-19-mediated ANE was described by Poyiadji et al. in a 50-year-old female patient who presented with a 3-day history of fever, cough, and altered mental status. Magnetic resonance imaging (MRI) of the brain demonstrated hemorrhagic rim-enhancing lesions within the medial temporal lobes, subinsular regions, and bilateral thalami. The intravenous immunoglobulin (IVIG) injection was administrated for therapy. Therefore, it could be induced that the cytokine storm syndrome has frequently been observed in patients with COVID-19 that might play a leading role in ANE development (Poyiadji et al. 2020). Dixon et al. reported another case of hemorrhagic ANE with early involvement of brain stem, which is possibly associated with COVID-19. A 59-year-old female with a history of transfusion-dependent aplastic anemia presented with reduced consciousness and seizures 10 days after the initiation of COVID-19 symptoms. The patient was positive for SARS-CoV-2 as proved by nasopharyngeal swat testing but cerebrospinal fluid (CSF) PCR for SARS-CoV-2 was negative. By the sixth day, MRI 
revealed progressive swelling of the brain stem accompanied by symmetrical hemorrhagic lesions of the brain stem, putamina, thalamic nuclei, and amygdalae. The patient did not respond well to treatment with steroids and died after eight days of admission (Dixon et al. 2020). Afshar et al. reported a 39-year-old female presenting with fever, anorexia, drowsiness, dry cough, and myalgia. Although the patient tested negative for COVID-19 in the nasopharyngeal swab and CSF, she was diagnosed with COVID-19 based on the chest $\mathrm{CT}$ and the clinical laboratory along with imaging findings were compatible with parainfectious encephalitis (Afshar et al. 2020). She maintained IgG- and IgM-positive status during the admission. The patient received a 3-day IVIG therapy before diagnosis of neurologic involvement, and IVIG therapy was sustained predicated on improved clinical and radiological results. However, following the emergence of the headaches, IVIG therapy was stopped, and the patient was managed with methylprednisolone, which resulted in complete resolution of neurological symptoms. The patient was fully asymptomatic upon hospital discharge (Afshar et al. 2020). Based on the aforementioned reported evidence, COVID-19 might be related to severe acute encephalopathy (Dixon et al. 2020).

\section{Meningoencephalitis}

The first case of SARS-CoV-2-associated meningoencephalitis was reported in a 24-year-old male presenting with fever, generalized fatigue, unconsciousness, neck stiffness, and seizures during admission. The patient tested positive for COVID-19 in the CSF, while the nasopharyngeal swab yielded negative results. Brain MRI suggested meningoencephalitis, demonstrating hyperintensity in the wall of the right lateral ventricle wall, right mesial temporal lobe, and hippocampus (Moriguchi et al. 2020). Furthermore, Duong et al. reported meningoencephalitis in a 41-year-old woman who was positive for COVID-19 and complained about neck stiffness, fever, and pleocytosis (Duong et al. 2020).

\section{Seizures}

Seizures are usually caused either following primary viral infection or via restimulation of the latent viral infections (Karimi et al. 2020). SARS-CoV-2-infected patients have occasionally been presented with seizures (Mao et al. 2020). Mao et al. reported that $0.5 \%$ of COVID-19 patients suffered from seizures (Mao et al. 2020). In a multicenter retrospective study of 304 COVID-19 cases with no background history of seizures, only two patients showed seizure-like symptoms associated with hypocalcemia, suggesting a minimal risk of seizure development during acute COVID-19 illness (Lu et al. 2020). Furthermore, Karimi et al. described the first case of frequent seizures in association with COVID-19 in a 30-year-old woman, with no medical history. The patient was presented with 5-day dry cough followed by fever and fatigue, and 2 days prior to admission, she had experienced a generalized tonic-clonic seizure for the first time. Nasopharyngeal swab for COVID-19 infection was positive; nevertheless, the CSF sample was unremarkable for SARS$\mathrm{CoV}-2$. She was treated with intravenous phenytoin and levetiracetam (Karimi et al. 2020).

\section{Acute cerebrovascular disease}

Apart from respiratory complications, one of the neurological manifestations that were reported in some of the COVID19 patients is acute cerebrovascular disease (ACVD). Mao et al. demonstrated that $5.7 \%$ of severe COVID-19 cases developed ACVD, which was commonly presented as a stroke, with ischemic stroke (IS) being prevalent than hemorrhagic stroke (HS) (Mao et al. 2020). In a retrospective investigation on 304 COVID-19 patients, $27 \%$ of the cases were reported to develop an IS throughout the infection $(\mathrm{Lu}$ et al. 2020). In another study on 184 patients, three cases were described to be affected by an IS (Klok et al. 2020).

Another retrospective study implied that $10.8 \%$ of 92 patients who died due to the COVID-19 developed a cerebrovascular event (Yang et al. 2020). COVID-19 poses a risk of IS, most probably through excessive inflammation inducement, hypoxic conditions, immobilization, or diffuse intravascular coagulation (Klok et al. 2020). Li et al. suggested the presumption that elderly patients are more vulnerable to ACVD development as they observed the ACVD following the COVID-19 infection in eleven elderly patients (Li et al. 2020a). Moreover, Avula et al. reported that four patients who had initially experienced a stroke later tested positive for SARS-CoV-2 (Avula et al. 2020). Al Saiegh et al. presented two cases with confirmed SARS-CoV-2 infection who had concurrent neurological symptoms while repeatedly testing negative for the virus RNA in their CSF. In the first case, a 31-year-old male without any hypertension or other chronic illness background presented with acute subarachnoid hemorrhage and later tested positive for SARS-CoV-2. In the second case, a 62-year-old female developed an IS with hemorrhagic conversion without any of the SARS-CoV-2 infection-associated symptoms and tested positive for SARS-CoV-2 soon after (Al Saiegh et al. 2020).

As it was evident in the so-called studies and based on the report by Neumann et al. (Neumann et al. 2020), CSF PCR testing for SARS-CoV-2 is usually negative in patients with neurological complications. However, like other viral brain infections, a negative result by RT-PCR does not exclude the viral presence in the brain tissue. Therefore, additional studies on SARS-CoV2 antibodies in CSF would be beneficial (Neumann et al. 2020). In a study by Sharifi et al., three adult patients were described with IS associated with 
COVID-19 infection. All patients were presented with severe life-threatening symptoms and were mechanically ventilated in the intensive care unit (ICU). The exact mechanism of stroke subsequent to viral infection is yet enigmatic. One of the proposed mechanisms is that the vascular wall is probably disturbed during viral infection, either directly or indirectly, via the secretion of pro-inflammatory cytokines, which then lead to the proliferation of smooth muscle cells and platelet aggregation (Sharifi-Razavi et al. 2020). Interestingly, Yaghi et al. aimed to compare the clinical presentations of stroke patients diagnosed with COVID-19, to those without COVID-19. They observed a relatively low rate of imaging-confirmed IS in hospitalized COVID-19 patients. In COVID-19 patients with IS, most strokes were considered cryptogenic, possibly due to the acquired hypercoagulability, and were associated with high mortality. Further data is required to assess the efficacy of anticoagulation therapy for stroke and other thrombotic event prevention in COVID-19 patients (Yaghi et al. 2020).

\section{PNS manifestations}

\section{Guillain-Barré syndrome}

GBS is an acute immune-related neurologic disease (polyradiculoneuropathy) that is usually elicited by various infections (Sedaghat and Karimi 2020). The mechanism underlying GBS is that the molecular mimicry between certain viral proteins and peripheral nerve proteins such as gangliosides can cause the host immune system to mistakenly attack the adjacent peripheral nerves by damaging their myelin insulation or axons (Guidon and Amato 2020). It is not yet clear whether SARS-CoV-2 infection triggers the expression of anti-ganglioside antibodies that normally coincide with certain GBS forms (Sedaghat and Karimi 2020).

Based on recently published reports, an increase in the number of GBS cases following the COVID-19 suggests a possible correlation. The first case of COVID-19-related GBS was described by Zhao et al. in a 61-year-old female with acute progressive lower limb weakness who had traveled to China. She was diagnosed with GBS and was given IVIG and antiviral administration. She showed classic symptoms of COVID-19 by the eighth day and tested positive later on. Following the therapy and isolation, she eventually demonstrated an acceptable motor recovery (Zhao et al. 2020).

In another similar case observed by Virani et al., a 54-yearold male suffered from weakness and numbness of his lower extremities. The patient also had fever, cough, and diarrhea and tested positive for SARS-CoV-2. Due to acute polyneuropathy diagnosis, he received therapy with IVIG, and responded well and was finally subjected to physical therapy (Virani et al. 2020). A more thorough report regarding the association between the GBS and COVID-19 described five COVID-19 cases in Italy. Based on these findings, the main neurological symptoms included paresthesia and lower-limb weakness. Nasopharyngeal swabs for SARS-CoV-2 were positive in four patients at the beginning of the neurologic manifestations. In contrast, the other patient was negative for a nasopharyngeal swab and bronchoalveolar lavage tests and later was positive by serologic test. It is worth mentioning that the CSF test for SARS-CoV-2 was negative for all of the cases, and all five patients received IVIG treatment (Toscano et al. 2020).

Mozhdehipanah et al. described four patients (three females, one male mean age 43) who developed GBS symptoms 21 to 30 days after the onset of COVID-19 symptoms. Of these cases, three patients suffered from paresthesia and lower-limb weakness, and one presented with paresthesia and facial paresis, who subsequently developed mild lower-limb weakness accompanied by autonomic instability. Treatment was managed with IVIG therapy in three patients and one with a course of therapeutic plasma exchange (TPE) (Mozhdehipanah et al. 2020). Later, Sedaghat and Karimi described a 65-year-old male presented with progressive ascending quadriparesis and bilateral facial paralysis following 2 weeks of the SARS-CoV-2 infection. The patient received five days of IVIG administration (Sedaghat and Karimi 2020). In another report, Ebrahimzadeh et al. presented two COVID-19 cases associated with GBS (Ebrahimzadeh et al. 2020). Case 1 was a 46-year-old man who complained about the pain and numbness in the distal upper and lower extremities for six days. The common symptoms of COVID-19 were revealed eighteen days before the onset of neurological symptoms as confirmed by a highresolution CT scan (HRCT) of the chest and nasopharyngeal sample PCR. Case 2 was a 65 -year-old male who developed paresthesia and progressive ascending weakness in the upper and lower extremities 10 days after COVID-19 diagnosis (validated by HRCT and nasopharyngeal sample PCR). Although the vital mechanism underlying GBS development in COVID-19 cases is not yet fully described, it was hypothesized that SARS-CoV-2 primes the inflammatory cells to produce and secrete a series of pro-inflammatory cytokines, which subsequently initiate immune-associated polyneuropathy (McGonagle et al. 2020). However, further investigations are exceedingly required regarding the mechanism of GBS formation in COVID-19 patients.

\section{Miller Fisher syndrome}

As a possible variant of the GBS, Miller Fisher syndrome (MFS) is clinically defined by a triad of symptoms, including ataxia, areflexia, and ophthalmoplegia. COVID-19 patients were also reported to experience other symptoms relating to this triad, such as astasia, trunk ataxia, and dysplasia (de Goés et al. 2020). Gutiérrez-Ortiz et al. reported two COVID-19 patients presented with acute MFS and polyneuritis cranialis, respectively. Case 1 
received immunoglobulin therapy, and case 2 was treated with acetaminophen; both satisfactorily progress. It was concluded that neurological symptoms could occur due to an abnormal immune response to SARS-CoV-2. However, the clinical spectrum of neurological symptoms regarding the COVID-19 has to be fully elucidated (Gutiérrez-Ortiz et al. 2020).

\section{Myasthenia gravis}

Restivo et al. described three patients without previous neurologic or autoimmune disorders who were acetylcholine receptors (AChR) antibody-positive MG after the onset of COVID-19. Two male patients initially had a fever and developed muscular fatigability and diplopia a few days later. Nasopharyngeal swab testing for SARS-CoV-2 was positive for both patients. The third patient was a female who had fever and cough at the disease onset and developed bilateral ocular ptosis, diplopia, and hypophonia 5 days later. Nasopharyngeal swab testing of COVID-19 was negative for the first time but yielded positive in the second test a few days later. One patient was treated with pyridostigmine bromide and prednisone, the second one received a cycle of IVIG administration, and the third one was given hydroxychloroquine (Restivo et al. 2020).

In an observational retrospective study, Camelo-Filho et al. described clinical characteristics and outcomes of 15 hospitalized patients presented with diagnosed MG (based on antibodies or electrophysiological findings) and concurrent COVID-19 infection. One patient was treated with IVIG, and four patients with plasma exchange therapy (PLEX). Six patients underwent continuous neuromuscular blocking agents (NMBs) for mechanical ventilation (MV), four of whom were deceased, and one was still hospitalized and mechanically ventilated (Camelo-Filho et al. 2020). Moreover, Sriwastava et al. reported the first case of ocular myasthenia gravis (OMG) developing concurrently with SARS-CoV-2 infection in a 65-year-old female. Two weeks before being admitted to the hospital, the common symptoms of COVID19 were revealed and she tested positive for COVID-19 via a nasopharyngeal RT-PCR swab test. Based on the patient's history, laboratory tests, and electrodiagnostic findings, she was diagnosed with seropositive OMG and responded well to a standard pyridostigmine dose. These studies highlight the notion that the production of antibodies against neuromuscular junctions triggering MG could be a consequence of COVID-19 infection (Sriwastava et al. 2020).

\section{Anosmia and hypogeusia}

Anosmia and hypogeusia are often reported as symptoms of COVID-19, both of which may be the consequence of an aberrant immunological or neurological response preceding the respiratory symptoms (Kipnis 2018; Pallanti 2020). Bon et al. hypothesized that sudden hyposmia or anosmia related to COVID-19 might arise from the apoptosis of olfactory receptor neurons as a protective host defense mechanism. Since olfaction is the only sense with regenerative properties in adults, it seems logical to sacrifice smelling for neuroprotection (Le Bon and Horoi 2020). Brann et al. analyzed the expression of genes that are mediating the cell entry of SARS-CoV-2 (ACE2 and TMPRSS2) in olfactory mucosa to investigate the sudden anosmia associated with COVID19. As they described, ACE2 expression was sparsely observed in support cells, stem cells, and pericytes but not in olfactory nerves. Therefore, olfactory dysfunction is not directly originated from a viral infection of olfactory receptor neurons (ORNs), rather by a secondary inflammation or infection of olfactory neurons. Interestingly, they found that $\mathrm{CoV}$ infection of rat olfactory epithelial cells resulted in extensive loss of neurosensory cilia from ORNs, and limited infection of supporting cells. The evidence collectively suggests that the COVID-19-mediated olfactory disturbance is probably mediated by the immune defenses against SARSCoV-2 rather than direct viral damage (Brann et al. 2020). The effect of SARS-CoV-2 in the nasal cavity was first investigated by Bryche et al., who demonstrated olfactory loss and dysfunction in the SARS-COV-2-infected golden Syrian hamsters. By the second day of nasal instillation of SARS-CoV-2 particles, they detected extensive damage of the olfactory epithelial cells, leading to a massive lack of cilia essential for odor recognition. These complications were caused due to the infection of a significant number of sustentacular cells, and were not associated with olfactory nerves. Additionally, no virus was detected in the olfactory bulbs. Furthermore, massive infiltration of immune cells into the olfactory epithelium and lamina propria of infected animals was observed, which could be associated with desquamation of the olfactory epithelial tissue. The olfactory epithelium was restored in part after 14 days of infection. These findings partially explain the anosmia observed in COVID-19 patients, but need to be further validated by additional studies involving human olfactory epithelium (Bryche et al. 2020).

\section{Conclusion}

Like the rest of the world, Iran is experiencing an unprecedented massive outbreak of COVID-19 infections and according to statistics ranks 11 th in the number of total deaths due to COVID-19 as of February 16, 2021 (https:// www.worldometers.info/coronavirus/). Based on the epidemiological study of COVID-19 patients in Iran, elderly male patients who also have comorbidities are significantly associated with the risk of mortality among patients with 
COVID-19 (Nikpouraghdam et al. 2020). Respiratory manifestations are the most common features reported in patients who suffered from COVID-19; however, there are already reports of neurological complications in some cases. To date, our knowledge is still limited regarding the mechanisms and complications of newly emerged SARS-CoV-2 interacting with the nervous system. Therefore, meticulous investigation of COVID-19-associated neurological manifestations is a step toward the improved understanding of SARS-CoV-2 infection, designing novel intervention strategies based on disease severity, and avoiding further spread.

Abbreviations ACE2: angiotensin-converting enzyme 2; AChR: acetylcholine receptors; ACVD: acute cerebrovascular disease; ANE: acute necrotizing encephalopathy; APC: antigen-presenting cells; BBB: blood-brain barrier; CNS: central nervous system; COVID-19: coronavirus disease 2019; CSF: cerebrospinal fluid; ENS: enteric nervous system; GBS: Guillain-Barré Syndrome; GGO: ground-glass opacity; HIV: human immunodeficiency virus; HRCT: high-resolution CT scan; HS: hemorrhagic stroke; ICU: intensive care unit; IS: ischemic stroke; IVIG: intravenous immunoglobulin; IL-6: interleukin 6; MFS: Miller Fisher syndrome; MG: myasthenia gravis; MRI: magnetic resonance imaging; MV: mechanical ventilation; NMBs: neuromuscular blocking agents; OMG: ocular myasthenia gravis; ORNs: olfactory receptor neurons; PLEX: plasma exchange therapy; PNS: peripheral nervous system; SARS-CoV-2: severe acute respiratory syndrome coronavirus 2; TMPRSS2: transmembrane serine protease 2; TNF- $\alpha$ : tumor necrosis factor $\alpha$; TPE: therapeutic plasma exchange

\section{Declarations}

Conflict of interest The authors declare that they have no conflict of interest.

\section{References}

Afshar H, Yassin Z, Kalantari S, Aloosh O, Lotfi T, Moghaddasi M, Sadeghipour A, Emamikhah M (2020) Evolution and resolution of brain involvement associated with SARS-CoV2 infection: a close clinical-paraclinical follow up study of a case. Mult Scler Relat Disord: 102216

Ahmadirad N, Ghasemi Z (2020) COVID-19 and central nervous system: entry routes and probable damages. Basic Clin Neurosci 11:217-224

Al Saiegh F, Ghosh R, Leibold A, Avery MB, Schmidt RF, Theofanis T, Mouchtouris N, Philipp L, Peiper SC, Wang ZX (2020) Status of SARS-CoV-2 in cerebrospinal fluid of patients with COVID-19 and stroke. J Neurol Neurosurg Psychiatry

Avula A, Nalleballe K, Narula N, Sapozhnikov S, Dandu V, Toom S, Glaser A, Elsayegh D (2020) COVID-19 presenting as stroke. Brain Behav Immun

Baig AM, Khaleeq A, Ali U, Syeda H (2020) Evidence of the COVID-19 virus targeting the CNS: tissue distribution, hostvirus interaction, and proposed neurotropic mechanisms. ACS Chem Neurosci 11:995-998

Berger JR (2020) COVID-19 and the nervous system. J Neurovirol: 1
Bohmwald K, Galvez N, Ríos M, Kalergis AM (2018) Neurologic alterations due to respiratory virus infections. Front Cell Neurosci 12:386

Brann DH, Tsukahara T, Weinreb C, Lipovsek M, Van den Berge K, Gong B, Chance R, Macaulay IC, Chou HJ, Fletcher RB (2020) Non-neuronal expression of SARS-CoV-2 entry genes in the olfaory system suggests mechanisms underlying COVID19-associated anosmia. Sci Adv: eabc5801

Bryche B, St Albin A, Murri S, Lacôte S, Pulido C, Gouilh MA, Lesellier S, Servat A, Wasniewski M, Picard-Meyer E (2020) Massive transient damage of the olfactory epithelium associated with infection of sustentacular cells by SARS-CoV-2 in golden Syrian hamsters. Brain Behav Immun

Camelo-Filho AE, Silva A, Estephan EP, Zambon AA, Mendonça RH, Souza PV, Pinto WB, Oliveira AS, Dangoni-Filho I, Pouza AF (2020) Myasthenia gravis and COVID-19: clinical characteristics and outcomes. Front Neurol 11:1053

Chen R, Yu J, Wang K, Howard D, French L, Chen Z, Wen C, Xu Z (2020) The spatial and cell-type distribution of SARS-CoV-2 receptor ACE2 in human and mouse brain. BioRxiv

Collins AR (2002) In vitro detection of apoptosis in monocytes/ macrophages infected with human coronavirus. Clin Diagn Lab Immunol 9:1392-1395

Conde G, Pájaro LDQ, Marzola IDQ, Villegas YR, Salazar LRM (2020) Neurotropism of SARS-CoV 2: mechanisms and manifestations. J Neurol Sci

de Goés A, Cardoso B, Tavares F, do Monte R, Melo R, (2020) COVID-19 and nervous system: under estimated clinical and prognostic aspects. Int J Neurol Neurother 7:100

Desforges M, Miletti TC, Gagnon M, Talbot PJ (2007) Activation of human monocytes after infection by human coronavirus $229 \mathrm{E}$. Virus Res 130:228-240

Dixon L, Varley J, Gontsarova A, Mallon D, Tona F, Muir D, Luqmani A, Jenkins IH, Nicholas R, Jones B (2020) COVID19-related acute necrotizing encephalopathy with brain stem involvement in a patient with aplastic anemia. Neurol Neuroimmunol Neuroinflamm 7

Dubé M, Le Coupanec A, Wong AH, Rini JM, Desforges M, Talbot PJ (2018) Axonal transport enables neuron-to-neuron propagation of human coronavirus OC43. J Virol 92

Duong L, Xu P, Liu A (2020) Meningoencephalitis without respiratory failure in a young female patient with COVID-19 infection in Downtown Los Angeles, early April 2020. Brain Behav Immun

Ebrahimzadeh SA, Ghoreishi A, Rahimian N (2020) Guillain-Barré syndrome associated with the coronavirus disease 2019 (COVID19). Neurol Clin Pract

Esposito G, Capoccia E, Gigli S, Pesce M, Bruzzese E, D’Alessandro A, Cirillo C, Di Cerbo A, Cuomo R, Seguella L (2017) HIV-1 Tatinduced diarrhea evokes an enteric glia-dependent neuroinflammatory response in the central nervous system. Sci Rep 7:1-11

Esposito G, Pesce M, Seguella L, Sanseverino W, Lu J, Sarnelli G (2020) Can the enteric nervous system be an alternative entrance door in SARS-CoV2 neuroinvasion? Brain Behav Immun

Glass WG, Subbarao K, Murphy B, Murphy PM (2004) Mechanisms of host defense following severe acute respiratory syndrome-coronavirus (SARS-CoV) pulmonary infection of mice. J Immunol 173:4030-4039

Gowrisankar YV, Clark MA (2016) Angiotensin II regulation of angiotensin-converting enzymes in spontaneously hypertensive rat primary astrocyte cultures. J Neurochem 138:74-85

Guidon AC, Amato AA (2020) COVID-19 and neuromuscular disorders. Neurology 94:959-969

Gutiérrez-Ortiz C, Méndez A, Rodrigo-Rey S, San Pedro-Murillo E, Bermejo-Guerrero L, Gordo-Mañas R, de Aragón-Gómez F, Benito-León J (2020) Miller Fisher syndrome and polyneuritis cranialis in COVID-19. Neurology 
Huang J, Zheng M, Tang X, Chen Y, Tong A, Zhou L (2020) Potential of SARS-CoV-2 to cause CNS infection: biologic fundamental and clinical experience. Front Neurol 11:659

II PAS, McGavern DB (2015) Portals of viral entry into the central nervous system. The Blood-Brain Barrier in Health and Disease, Volume Two: Pathophysiology and Pathology 23

Jacomy H, Fragoso G, Almazan G, Mushynski WE, Talbot PJ (2006) Human coronavirus OC43 infection induces chronic encephalitis leading to disabilities in BALB/C mice. Virology 349:335-346

Jacomy H, Talbot PJ (2003) Vacuolating encephalitis in mice infected by human coronavirus OC43. Virology 315:20-33

Karimi N, Sharifi Razavi A, Rouhani N (2020) Frequent convulsive seizures in an adult patient with COVID-19: a case report. Iran Red Crescent Med J

Kipnis J (2018) Immune system: the "seventh sense." J Exp Med 215:397

Klok F, Kruip M, Van der Meer N, Arbous M, Gommers D, Kant K, Kaptein F, van Paassen J, Stals M, Huisman M (2020) Incidence of thrombotic complications in critically ill ICU patients with COVID-19. Thromb Res

Lavi E, Cong L (2020) Type I astrocytes and microglia induce a cytokine response in an encephalitic murine coronavirus infection. Exp Mol Pathol: 104474

Le Bon S, Horoi M (2020) Is anosmia the price to pay in an immuneinduced scorched-earth policy against COVID-19? Med Hypotheses: 109881

Li Y, Wang M, Zhou Y, Chang J, Xian Y, Mao L, Hong C, Chen S, Wang Y, Wang H (2020a) Acute cerebrovascular disease following COVID-19: a single center, retrospective, observational study

Li YC, Bai WZ, Hashikawa T (2020) The neuroinvasive potential of SARS-CoV2 may play a role in the respiratory failure of COVID19 patients. J Med Virol 92:552-555

Lu L, Xiong W, Liu D, Liu J, Yang D, Li N, Mu J, Guo J, Li W, Wang G (2020) New onset acute symptomatic seizure and risk factors in coronavirus disease 2019: a retrospective multicenter study. Epilepsia

Mao L, Jin H, Wang M, Hu Y, Chen S, He Q, Chang J, Hong C, Zhou Y, Wang D (2020) Neurologic manifestations of hospitalized patients with coronavirus disease 2019 in Wuhan, China. JAMA neurology 77:683-690

McCray PB, Pewe L, Wohlford-Lenane C, Hickey M, Manzel L, Shi L, Netland J, Jia HP, Halabi C, Sigmund CD (2007) Lethal infection of K18-hACE2 mice infected with severe acute respiratory syndrome coronavirus. J Virol 81:813-821

McGavern DB, Kang SS (2011) Illuminating viral infections in the nervous system. Nat Rev Immunol 11:318-329

McGonagle D, Sharif K, O’Regan A, Bridgewood C (2020) Interleukin-6 use in COVID-19 pneumonia related macrophage activation syndrome. Autoimmun Rev: 102537

Montalvan V, Lee J, Bueso T, De Toledo J, Rivas K (2020) Neurological manifestations of COVID-19 and other coronavirus infections: a systematic review. Clin Neurol Neurosurg 194:105921

Moriguchi T, Harii N, Goto J, Harada D, Sugawara H, Takamino J, Ueno M, Sakata H, Kondo K, Myose N (2020) A first case of meningitis/encephalitis associated with SARS-Coronavirus-2. Int J Infect Dis

Mozhdehipanah H, Paybast S, Gorji R (2020) Guillain-Barré syndrome as a neurological complication of COVID-19 infection: a case series and review of the literature. ICNSJ 7:156-161

Natoli S, Oliveira V, Calabresi P, Maia LF, Pisani A (2020) Does SARS-Cov-2 invade the brain? Translational lessons from animal models. Eur J Neurol

Netland J, Meyerholz DK, Moore S, Cassell M, Perlman S (2008) Severe acute respiratory syndrome coronavirus infection causes neuronal death in the absence of encephalitis in mice transgenic for human ACE2. J Virol 82:7264-7275

Neumann B, Schmidbauer ML, Dimitriadis K, Otto S, Knier B, Niesen WD, Hosp JA, Günther A, Lindemann S, Nagy G (2020) Cerebrospinal fluid findings in COVID-19 patients with neurological symptoms. J Neurol Sci 418

Nikpouraghdam M, Farahani AJ, Alishiri G, Heydari S, Ebrahimnia M, Samadinia H, Sepandi M, Jafari NJ, Izadi M, Qazvini A (2020) Epidemiological characteristics of coronavirus disease 2019 (COVID-19) patients in IRAN: a single center study. J Clin Virol 127:104378

Pallanti S (2020) Importance of SARs-Cov-2 anosmia: from phenomenology to neurobiology. Compr Psychiatry: 152184

Paniz-Mondolfi A, Bryce C, Grimes Z, Gordon RE, Reidy J, Lednicky J, Sordillo EM, Fowkes M (2020) Central nervous system involvement by severe acute respiratory syndrome coronavirus-2 (SARSCoV-2). J Med Virol 92:699-702

Perlman S, Evans G, Afifi A (1990) Effect of olfactory bulb ablation on spread of a neurotropic coronavirus into the mouse brain. J Exp Med 172:1127-1132

Poyiadji N, Shahin G, Noujaim D, Stone M, Patel S, Griffith B (2020) COVID-19-associated acute hemorrhagic necrotizing encephalopathy: CT and MRI features. Radiology: 201187

Restivo DA, Centonze D, Alesina A, Marchese-Ragona R (2020) Myasthenia gravis associated with SARS-CoV-2 infection. Ann Intern Med

Sedaghat Z, Karimi N (2020) Guillain Barre syndrome associated with COVID-19 infection: a case report. J Clin Neurosci

Sharifi-Razavi A, Karimi N, Zarvani A, Cheraghmakani H, Baghbanian SM (2020) Ischemic stroke associated with novel coronavirus 2019: a report of three cases. Int J Neurosci: 1-5

Sriwastava S, Tandon M, Kataria S, Daimee M, Sultan S (2020) New onset of ocular myasthenia gravis in a patient with COVID-19: a novel case report and literature review. J Neurol: 1-7

St-Jean JR, Jacomy H, Desforges M, Vabret A, Freymuth F, Talbot PJ (2004) Human respiratory coronavirus OC43: genetic stability and neuroinvasion. J Virol 78:8824-8834

Swanson PA II, McGavern DB (2015) Viral diseases of the central nervous system. Curr Opin Virol 11:44-54

Toscano G, Palmerini F, Ravaglia S, Ruiz L, Invernizzi P, Cuzzoni MG, Franciotta D, Baldanti F, Daturi R, Postorino P (2020). GuillainBarré syndrome associated with SARS-CoV-2. N Engl J Med

Virani A, Rabold E, Hanson T, Haag A, Elrufay R, Cheema T, Balaan M, Bhanot N (2020) Guillain-Barré syndrome associated with SARS-CoV-2 infection. IDCases: e00771

Wang Q, Zhang Y, Wu L, Niu S, Song C, Zhang Z, Lu G, Qiao C, Hu Y, Yuen KY (2020) Structural and functional basis of SARSCoV-2 entry by using human ACE2. Cell

Wentworth DE, Tresnan D, Turner B, Lerman I, Bullis B, Hemmila E, Levis R, Shapiro L, Holmes KV (2005) Cells of human aminopeptidase N (CD13) transgenic mice are infected by human coronavirus-229E in vitro, but not in vivo. Virology 335:185-197

Wheeler DL, Sariol A, Meyerholz DK, Perlman S (2018) Microglia are required for protection against lethal coronavirus encephalitis in mice. J Clin Investig 128:931-943

Xia H, Lazartigues E (2010) Angiotensin-converting enzyme 2: central regulator for cardiovascular function. Curr Hypertens Rep 12:170-175

Yaghi S, Ishida K, Torres J, Mac Grory B, Raz E, Humbert K, Henninger N, Trivedi T, Lillemoe K, Alam S (2020) SARS2CoV-2 and stroke in a New York healthcare system. Stroke: STROKEAHA. 120.030335

Yang F, Shi S, Zhu J, Shi J, Dai K, Chen X (2020) Analysis of 92 deceased patients with COVID-19. J Med Virol 
Zhao H, Shen D, Zhou H, Liu J, Chen S (2020) Guillain-Barré syndrome associated with SARS-CoV-2 infection: causality or coincidence? Lancet Neurol 19:383-384

Zubair AS, McAlpine LS, Gardin T, Farhadian S, Kuruvilla DE, Spudich S (2020) Neuropathogenesis and neurologic manifestations of the coronaviruses in the age of coronavirus disease 2019: a review. JAMA Neurol

Publisher's Note Springer Nature remains neutral with regard to jurisdictional claims in published maps and institutional affiliations. 